\title{
Primary pain generator identification by CT-SPECT in patients with degenerative spinal disease
}

\author{
Gabriel C. Tender, MD,, Caroline Davidson, MD, ${ }^{1}$ Jessica Shields, MD, ${ }^{1}$ Jared Robichaux, MD, ${ }^{1}$ \\ Joe Park, MD, ${ }^{2}$ Clifford L. Crutcher, MD, ${ }^{1}$ and Anthony M. DiGiorgio, DO, MHA ${ }^{1}$
}

Departments of ${ }^{1}$ Neurosurgery and ${ }^{2}$ Radiology, Louisiana State University Health Sciences Center, New Orleans, Louisiana

OBJECTIVE Axial spinal pain generators are difficult to identify using current diagnostic modalities. Merging CT with SPECT (CT-SPECT) scans allows for accurate identification of areas with increased osteoblastic activity, which may reflect pain generators. In this study, the authors aimed to evaluate the degree of pain improvement in patients who underwent surgery, addressing primary pain generators identified by CT-SPECT.

METHODS The authors retrospectively reviewed all patients with chronic axial spine pain who underwent diagnostic CT-SPECT at their institution and analyzed pain improvement in those who underwent surgical treatment in order to determine whether CT-SPECT correctly identified the primary pain generator.

RESULTS A total of 315 patients underwent diagnostic CT-SPECT between January 2014 and August 2018. Fortyeight patients underwent either cervical or lumbar fusion; there were 26 women ( 16 cervical, 10 lumbar) and 22 men (9 cervical, 13 lumbar). The overall axial spinal pain, as assessed through self-reporting of visual analog scale scores at 6 months postoperatively, improved from $9.04 \pm 1.4$ to $4.34 \pm 2.3(p=0.026)$, with cervical fusion patients improving from $8.8 \pm 1.8$ to $3.92 \pm 2.2(p=0.019)$ and lumbar fusion patients improving from $9.35 \pm 0.7$ to $4.87 \pm 2.3(p=0.008)$.

CONCLUSIONS CT-SPECT may offer a diagnostic advantage over current imaging modalities in identifying the primary pain generator in patients with axial spinal pain.

https://thejns.org/doi/abs/10.3171/2019.9.FOCUS19608

KEYWORDS CT-SPECT; spinal fusion; spine; pain

$\mathrm{C}$ HRONIC pain of spinal origin due to degenerative disease is common. The AANS/CNS guidelines on intractable low-back pain recommend a fusion procedure for axial pain due to 1- or 2-level degenerative disease that is refractory to conservative management. ${ }^{5}$ However, identifying the primary pain generator in these patients is notoriously difficult. Many imaging techniques and invasive tests have been tried to reliably identify pain generators in these challenging cases but with little success.

SPECT uses detection of ${ }^{99 \mathrm{~m}}$ technetium bound to osteoblasts to gain information on the amount of bone remodeling activity in the spinal axis. ${ }^{21}$ Using image-merging software between the SPECT and CT (CT-SPECT), we can thus identify, with a high degree of anatomical preci- sion, which parts of the spine exhibit increased osteoblastic activity. If this activity is increased around a joint (e.g., disc or facet joint), it may be indicative of a primary pain generator. Previous reports regarding the reliability of this imaging modality in identifying the pain generator have shown positive results. ${ }^{20}$ In the present study, we evaluated the degree of pain improvement in patients who underwent fusion surgery, addressing primary pain generators identified by CT-SPECT.

\section{Methods}

This is a retrospective study of all patients who underwent CT-SPECT at our institution between January 2014 (when we began using CT-SPECT as a diagnostic tool for 
spinal diseases) and August 2018. The study was approved by our institutional review board. Patient consent was not needed, as CT-SPECT has minimal risks and is considered routine at our institution. CT-SPECT at our institution is performed in accordance with the national guidelines. ${ }^{1}$

The patients were offered diagnostic CT-SPECT if they fulfilled the following criteria: ${ }^{8}$

- Severe chronic pain of suspected spinal origin (at least 6 of 10 on the visual analog scale [VAS]).

- Age 18-80 years.

- Pain duration at least 2 years.

- The patient must have had unsuccessful nonsurgical treatment for at least 1 year, with at least 3 months of physical therapy.

- The treating surgeon should interpret the pain as potentially emanating from one or more of the spinal segments.

- Radiological evidence (MRI, CT, and/or dynamic/scoliosis radiography) of degenerative changes (i.e., spondylosis) but without a clear identification of the primary pain generator (e.g., spondylolisthesis). The lack of imaging evidence of a primary pain generator included instability, as well as other, more subtle findings, such as loss of disc height, Modic changes, large osteophytes, facet hypertrophy, and so on. We considered unstable a segmental displacement of $3 \mathrm{~mm}$ or more on flexion-extension radiographs or on standing radiographs when compared to the MR images (taken in the supine position). These criteria are in line with the Medicare guidelines. Occasionally, patients with instability at one level, usually L4 -5 , had advanced degeneration at the adjacent level, usually L5-S1. Some of our patients underwent CT-SPECT to determine whether the degenerated (L5-S1) segment should be included in the fusion, along with the unstable (L4-5) level.

- Good understanding of English language.

The exclusion criteria were as follows:

- Psychiatric illness or evidence of emotional instability.

- Specific radiological findings, such as fractures, infection, inflammatory process, or neoplasm.

- Obvious painful and disabling arthritic hip and/or shoulder joints.

- Involvement in Workers' Compensation or litigation.

Pain-management procedures (e.g., epidural steroid injections, medial branch blocks, radiofrequency ablations, selective nerve root blocks) were occasionally used for diagnostic and/or treatment purposes. However, an attempt was made to avoid steroid injections at least 2 weeks prior to the CT-SPECT, since we do not know how, or even if, steroids affect osteoblast distribution.

Our treatment algorithm based on CT-SPECT results is as follows (Fig. 1): Patients with significantly increased technetium uptake in the cervical facet joints (1 or 2 adjacent levels) were offered a targeted medial branch block versus a minimally invasive facet fusion. Patients with significantly increased uptake in the cervical or lumbar disc joints (1 or 2 adjacent levels) were potentially offered a cervical or lumbar instrumented fusion, respectively. Patients with increased uptake in the lumbar facet joints, but without instability at the respective levels, were of- fered pain management only (no surgery). Patients who had negative CT-SPECT results, patients with more than 2 anatomical areas of significantly increased uptake, and patients who were offered but refused surgery were referred to pain management.

Our typical postoperative evaluation schedule involves clinic visits at 2 weeks, 2 months, 6 months, and 1 year, but most visits were completed per the aforementioned protocol \pm 4 weeks. The clinical outcome was determined based on patients' self-reported VAS scores for neck/lowback pain, as well as arm/leg pain, at 6 months postoperatively. Radiographic fusion was evaluated by CT at 1 year postoperatively.

All mean values are reported \pm SD. Standard two-tailed t-tests were used to compare the patients from pre- to postoperative scoring.

\section{Results}

We retrospectively identified a total of 315 patients who underwent CT-SPECT for degenerative spinal disease at our institution. Due to poor patient follow-up in our population, we focused on the 6-month follow-up results. Our 1 -year follow-up rate was $50 \%$. In these remaining patients, the pain scores remained similar to those seen at the 6-month follow-up visit. These results were not included in our final analysis, however.

Of these, 126 patients had negative CT-SPECT results (Fig. 2) and 103 had multiple areas of increased osteoblastic activity (Fig. 3), and therefore were deemed nonsurgical. Somewhat surprisingly, we occasionally found that some of the degenerated discs showed no increased technetium uptake on the CT-SPECT scan, which argued against performing fusion in these patients. Thirty-eight patients had potentially operative pathology demonstrated on CT-SPECT (Fig. 4), but they elected not to undergo a surgical intervention. These patients were referred for pain management.

The remaining 48 patients underwent either a cervical or lumbar fusion, based on the primary pain generator identified by CT-SPECT. There were 26 women (16 cervical, 10 lumbar) and 22 men (9 cervical, 13 lumbar), whose mean age was $53.9 \pm 8.9$ years (range $30-72$ years) (Table 1). Overall, the axial spinal pain, as assessed by self-reported VAS scores, improved from $9.04 \pm 1.4$ to $4.34 \pm 2.3(\mathrm{p}=0.026)$.

In the 25 patients who underwent cervical fusion (Table 2), the VAS score for axial pain decreased from $8.8 \pm 1.8$ preoperatively to $3.92 \pm 2.2$ at 6 months postoperatively (p $=0.019$ ) and for extremity pain from $7.28 \pm 2.9$ to $4.28 \pm$ $2.35(\mathrm{p}=0.09)$. Twelve of the 25 patients underwent CT scanning at 1 year postoperatively. All of these patients exhibited bone growth at the instrumented levels and no evidence of pseudarthrosis. There were no patients with increased or new pain postoperatively.

In the 23 patients who underwent lumbar fusion (Table 3 ), the VAS score for axial pain decreased from $9.35 \pm 0.7$ preoperatively to $4.87 \pm 2.3$ at 6 months postoperatively $(\mathrm{p}=0.008)$ and for extremity pain from $7.27 \pm 2.9$ to 4.46 $\pm 2.36(\mathrm{p}=0.009)$. Ten of the 23 patients underwent CT at 1 year postoperatively. All of these patients exhibited 


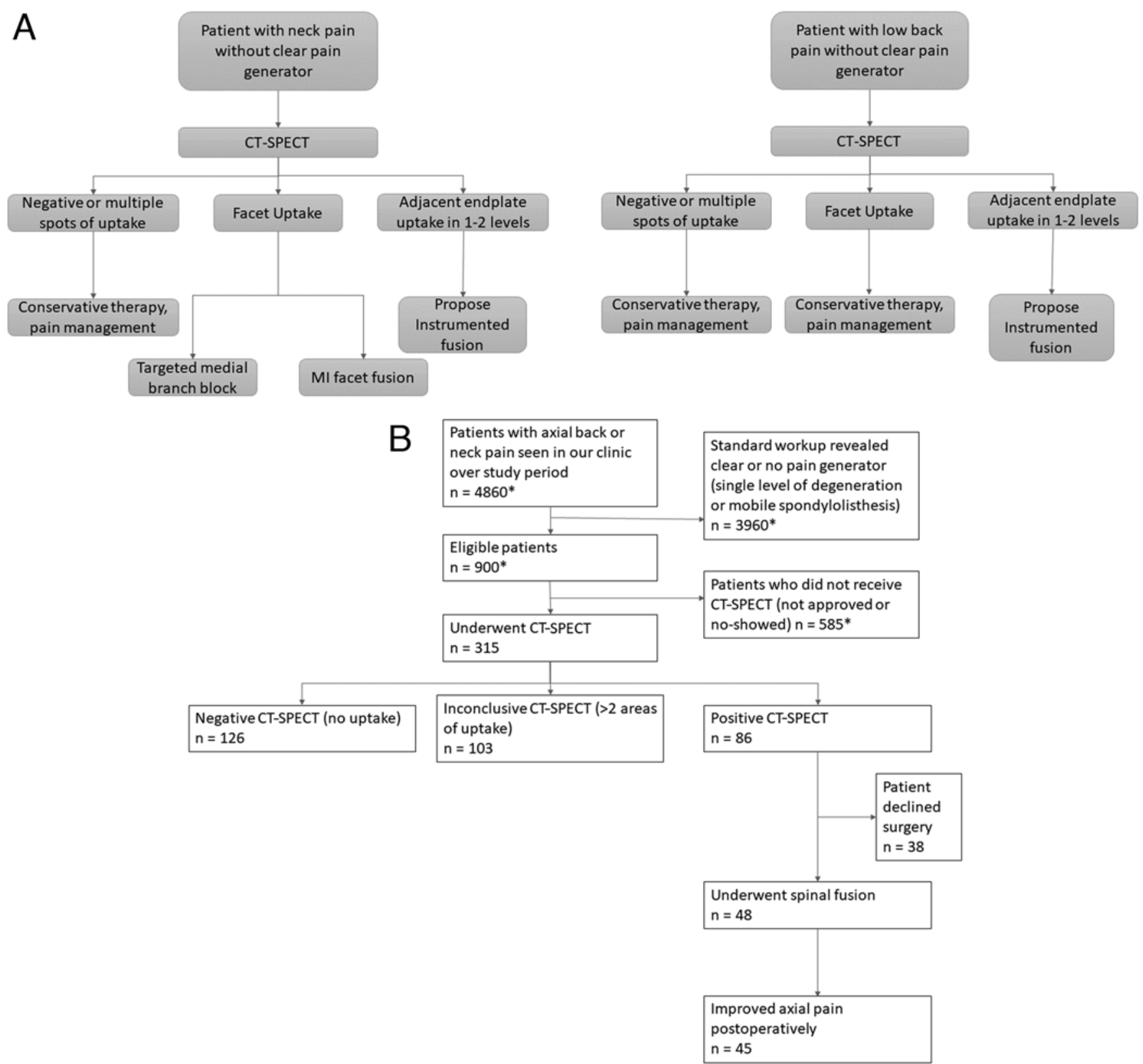

FIG. 1. A: Schematic representation of our treatment algorithms for patients with cervical (left) and lumbar (right) pain and no clear pain generator. B: Flow diagram for patient selection. Asterisks indicates the approximate number of patients. The mean number of patients seen in clinic every week is 100 , of which about 40 are new patients. Of these, about $75 \%$ (30 patients) have spinal problems; of these, about $90 \%$ (27 patients) have pain due to degenerative spinal disease, and about $15 \%$ (5 patients) are deemed to need identification of their primary pain generator by CT-SPECT. With a study period of 180 weeks, the approximate total number of patients with pain due to degenerative spinal disease is 4860 (27 patients $\times 180$ weeks $=4860$ patients), and the approximate number of patients eligible for CT-SPECT is 900 ( 5 patients $\times 180$ weeks $=900$ patients).

bone growth at the instrumented levels and no evidence of pseudarthrosis.

Three patients noted high pain scores postoperatively and were analyzed individually.

The patient in case 1 was a 60 -year-old man who underwent an L5-S1 minimally invasive (MI) transforaminal lumbar interbody fusion (TLIF), and his back and leg pain scores went from 9 and 8 preoperatively to 10 and 6 postoperatively, respectively. This was due to the fact that, while his preoperative axial low-back pain improved dramatically, about 2 months after surgery, he developed severe coccygeal pain. A lumbar CT scan at 6 months showed good placement of the instrumentation and some bone growth through the L5-S1 interspace but no coccygeal pathology. The patient was referred to pain management.

The patient in case 2 was a 61-year-old woman who noted back and leg pain scores of 8 and 1 preoperatively 


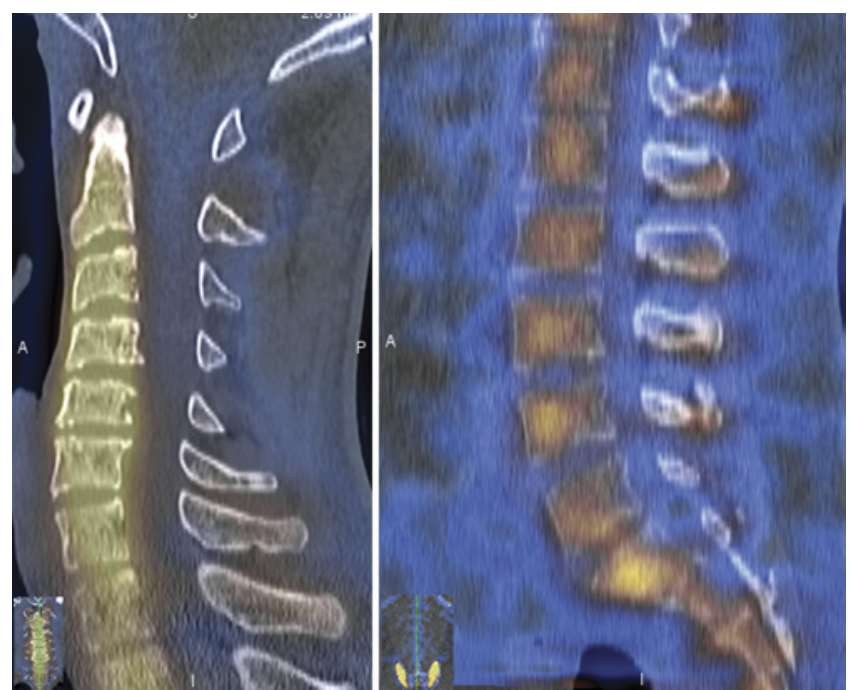

FIG. 2. Representative sagittal reconstruction (cervical [left] and lumbar [right]) CT-SPECT scans demonstrating physiological uptake in the vertebral bodies. The patients represented by these images were considered to have normal CT-SPECT findings with physiological uptake and were therefore deemed nonsurgical candidates.

that changed to 5 and 8 postoperatively, respectively. She underwent an L4-5 MI TLIF, with improvement in her preoperative axial and radicular pain, but a subsequent fall resulted in a right hip injury (hence, the extremity pain score of 8), prompting an orthopedic consultation. Postoperative lumbar imaging at 3 months showed no adverse findings.

The patient in case 3 was a 51-year-old homeless woman, and a smoker (stating she quit before surgery), who had undergone an L4-5 laminectomy 4 years prior to presentation to our clinic. CT-SPECT showed increased technetium uptake at L4-5 but also at L5-S1. The patient under-

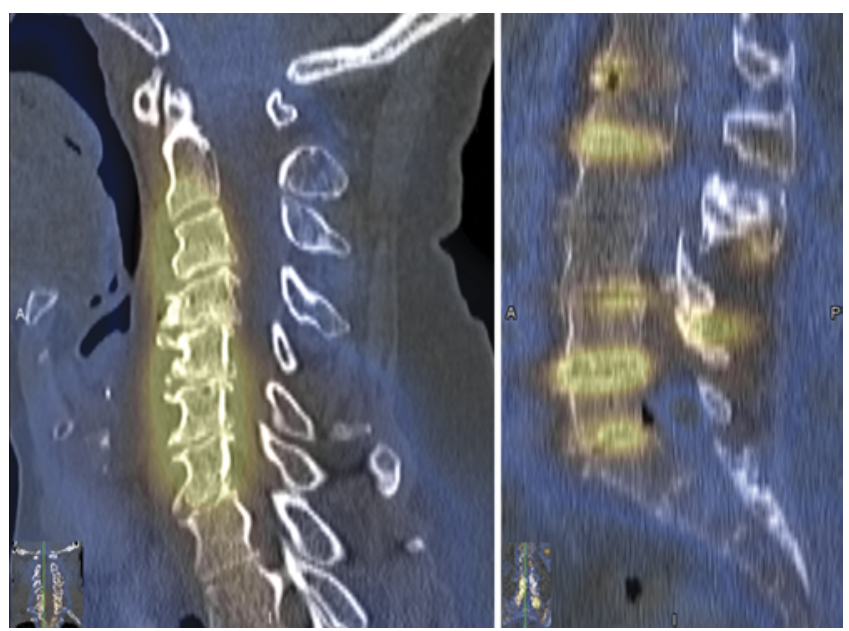

FIG. 3. Representative sagittal reconstruction (cervical [left] and lumbar [right]) CT-SPECT scans showing multiple areas of increased technetium uptake. The patients represented by these images were considered to have multiple pain generators and were therefore deemed nonsurgical candidates.
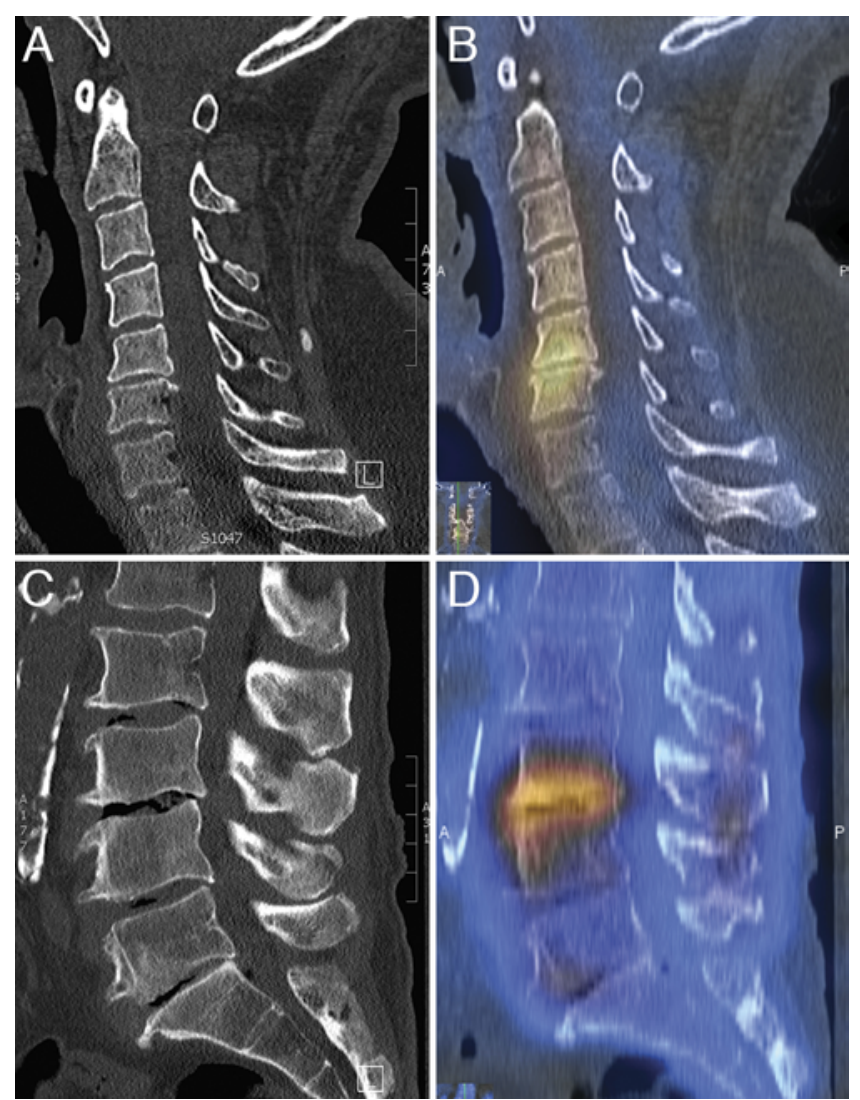

FIG. 4. Representative sagittal reconstruction (cervical CT [A], cervical CT-SPECT [B], lumbar CT [C], and lumbar CT-SPECT [D]) scans demonstrating increased technetium uptake in the endplates adjacent to a disc. The patients represented by these images underwent surgery at the positive levels and experienced good postoperative pain improvement.

went a TLIF at L4-5 and L5-S1. According to the patient, her preoperative symptoms did not improve (back and leg pain scores of 10 and 10 before surgery to 10 and 9 after surgery, respectively). A CT scan at 3 months postoperatively showed good placement of the instrumentation and no adverse effects. She was subsequently lost to follow-up.

\section{Discussion}

Identification of the primary pain generator in patients with chronic pain due to degenerative spine disease can be very difficult. Oftentimes, the patient's history and physical examination point to a spinal pain generator, but the typical imaging (dynamic radiography, MRI, and CT) fail to identify its exact location, in the absence of instability. ${ }^{16}$ Electromyography and nerve conduction velocity studies can help determining the spinal nerves affected, but they are of little use in patients with predominantly axial spinal pain. Invasive procedures, such as discograms and discoblocks, may be useful in detecting axial spinal pain generators, but they have their own significant risks and drawbacks. Additionally, the use of discograms is not endorsed by the AANS/CNS guidelines on treatment of degenerative spine disease. ${ }^{6}$

There has been increased interest in recent years to use 
TABLE 1. Demographics of patients who underwent surgery

\begin{tabular}{cc}
\hline Variable & Value \\
\hline Sex, no. & \\
\hline Male & $22^{*}$ \\
\hline Female & $26 \dagger$ \\
\hline Age (SD) & $53.9 \pm 8.9$ \\
\hline Mean & $30-72$ \\
\hline Range &
\end{tabular}

* There were 9 cervical and 13 lumbar fusions.

† There were 16 cervical and 10 lumbar fusions.

CT-SPECT in the diagnosis of axial spinal pain. SPECT was initially used for the diagnosis of metastases in cancer patients. ${ }^{4,7}$ Subsequently, the spine indications for SPECT expanded to include the evaluation of axial pain in young patients, $, 3,13$ as well as persistent pain in postoperative patients. ${ }^{14}$ The development of computer software to merge SPECT and CT scans has allowed for the precise anatomical localization of the areas with increased bone metabolism. The iliac bones and the vertebral bodies, particularly in young patients, have a naturally high concentration of osteoblasts and therefore often show a physiologically increased signal. However, increased osteoblastic activity around a joint (either disc or facet joint) may reflect the body's own attempt to autofuse that particular motion segment in order to stop the pain originating from that joint. The reliability of CT-SPECT in determining the primary spinal pain generator appears to be good. ${ }^{15,17,20}$ Moreover, it appears that CT-SPECT findings complement rather than reproduce MRI findings. ${ }^{12,18}$ Targeted treatments based on the CT-SPECT findings have shown positive results. ${ }^{10,11}$

We started using CT-SPECT as a diagnostic modality in 2014, and, after several successful cases, we started to incorporate it into our routine diagnostic workup for spine patients. ${ }^{19}$ Our results show that axial pain improved in most of the surgically treated patients and suggest that CTSPECT was correct in identifying the primary pain generator in these difficult patients, who could not be adequately diagnosed using the routine investigations. We have

TABLE 2. Summary of the surgical interventions in patients with cervical pain

\begin{tabular}{ll}
\hline \multicolumn{1}{c}{ Operation } & No. of Patients \\
\hline Anterior & \\
\hline Discectomy \& fusion & \\
\hline 1 level & 3 \\
\hline 2 levels & 6 \\
\hline 1-level corpectomy \& fusion & 1 \\
\hline 1-level discectomy \& arthroplasty & 1 \\
\hline Posterior & \\
\hline Minimally invasive fusion & 6 \\
\hline 1 level & 5 \\
\hline 2 levels & 3 \\
\hline 2-level open fusion
\end{tabular}

TABLE 3. Summary of the surgical interventions in patients with lumbar pain

\begin{tabular}{lc}
\hline \multicolumn{1}{c}{ Operation } & No. of Patients \\
\hline 1-level anterior discectomy \& fusion & 1 \\
\hline Lateral discectomy \& fusion & \\
\hline 1 level & 4 \\
\hline 2 levels & 3 \\
\hline Posterior discectomy \& fusion & \\
\hline 1 level & 8 \\
\hline 2 levels & 7 \\
\hline
\end{tabular}

also noticed some improvement in the extremity (arm or leg) pain, although statistical significance was not reached in the lower extremity. This improvement may imply that spinal nerves can be irritated at mobile painful joints, even without frank compression. Our results compare favorably with those published in the landmark article on spinal fusions, ${ }^{8}$ which reported good results in $63 \%$ of their surgically treated patients.

This is the first study, to our knowledge, analyzing pain improvement in surgical patients diagnosed by CTSPECT. Some of the limitations of this study include: 1) The cost of the CT-SPECT scan was not discussed. It is our understanding that this study is not covered by some of the health insurance companies and/or hospitals, which may limit its applications. 2) There was a relatively low percentage of patients who underwent surgical intervention based on the CT-SPECT results. The large number of negative CT-SPECT scans in our study may be perceived as wasteful; however, these patients were clearly diagnosed as nonoperative candidates and could be directed to other services, such as pain management. 3) There was no control group to use for results comparison.

\section{Conclusions}

This study suggests that CT-SPECT may offer a diagnostic advantage over the current modalities in identifying the primary pain generator in patients with pain of a degenerative spinal origin.

\section{References}

1. Bartel TB, Kuruva M, Gnanasegaran G, Beheshti M, Cohen EJ, Weissman AF, et al: SNMMI procedure standard for bone scintigraphy 4.0. J Nucl Med Technol 46:398-404, 2018

2. Bellah RD, Summerville DA, Treves ST, Micheli LJ: Lowback pain in adolescent athletes: detection of stress injury to the pars interarticularis with SPECT. Radiology 180:509_ 512,1991

3. Bodner RJ, Heyman S, Drummond DS, Gregg JR: The use of single photon emission computed tomography (SPECT) in the diagnosis of low-back pain in young patients. Spine (Phila Pa 1976) 13:1155-1160, 1988

4. Bushnell DL, Kahn D, Huston B, Bevering CG: Utility of SPECT imaging for determination of vertebral metastases in patients with known primary tumors. Skeletal Radiol 24:13-16, 1995

5. Eck JC, Sharan A, Ghogawala Z, Resnick DK, Watters WC 
III, Mummaneni PV, et al: Guideline update for the performance of fusion procedures for degenerative disease of the lumbar spine. Part 7: Lumbar fusion for intractable low-back pain without stenosis or spondylolisthesis. J Neurosurg Spine 21:42-47, 2014

6. Eck JC, Sharan A, Resnick DK, Watters WC III, Ghogawala Z, Dailey AT, et al: Guideline update for the performance of fusion procedures for degenerative disease of the lumbar spine. Part 6: Discography for patient selection. J Neurosurg Spine 21:37-41, 2014

7. Even-Sapir E, Martin RH, Barnes DC, Pringle CR, Iles SE, Mitchell MJ: Role of SPECT in differentiating malignant from benign lesions in the lower thoracic and lumbar vertebrae. Radiology 187:193-198, 1993

8. Fritzell P, Hägg O, Wessberg P, Nordwall A: 2001 Volvo Award winner in clinical studies: lumbar fusion versus nonsurgical treatment for chronic low back pain: a multicenter randomized controlled trial from the Swedish Lumbar Spine Study Group. Spine (Phila Pa 1976) 26:2521-2534, 2001

9. Han LJ, Au-Yong TK, Tong WC, Chu KS, Szeto LT, Wong $\mathrm{CP}$ : Comparison of bone single-photon emission tomography and planar imaging in the detection of vertebral metastases in patients with back pain. Eur J Nucl Med 25:635-638, 1998

10. Jain A, Jain S, Agarwal A, Gambhir S, Shamshery C, Agarwal A: Evaluation of efficacy of bone scan with SPECT/CT in the management of low back pain: a study supported by differential diagnostic local anesthetic blocks. Clin J Pain 31:1054-1059, 2015

11. Lee I, Budiawan H, Moon JY, Cheon GJ, Kim YC, Paeng JC, et al: The value of SPECT/CT in localizing pain site and prediction of treatment response in patients with chronic low back pain. J Korean Med Sci 29:1711-1716, 2014

12. Lehman VT, Murphy RC, Schenck LA, Carter RE, Johnson GB, Kotsenas AL, et al: Comparison of facet joint activity on 99mTc-MDP SPECT/CT with facet joint signal change on MRI with fat suppression. Diagn Interv Radiol 22:277-283, 2016

13. Lowe J, Schachner E, Hirschberg E, Shapiro Y, Libson E: Significance of bone scintigraphy in symptomatic spondylolysis. Spine (Phila Pa 1976) 9:653-655, 1984

14. Lusins JO, Danielski EF, Goldsmith SJ: Bone SPECT in patients with persistent back pain after lumbar spine surgery. J Nucl Med 30:490-496, 1989

15. Ravindra VM, Mazur MD, Bisson EF, Barton C, Shah LM, Dailey AT: The usefulness of single-photon emission computed tomography in defining painful upper cervical facet arthropathy. World Neurosurg 96:390-395, 2016

16. Resnick DK, Watters WC III, Sharan A, Mummaneni PV, Dailey AT, Wang JC, et al: Guideline update for the performance of fusion procedures for degenerative disease of the lumbar spine. Part 9: Lumbar fusion for stenosis with spondylolisthesis. J Neurosurg Spine 21:54-61, 2014
17. Russo VM, Dhawan RT, Baudracco I, Dharmarajah N, Lazzarino AI, Casey AT: Hybrid bone SPECT/CT imaging in evaluation of chronic low back pain: correlation with facet joint arthropathy. World Neurosurg 107:732-738, 2017

18. Russo VM, Dhawan RT, Dharmarajah N, Baudracco I, Lazzarino AI, Casey AT: Hybrid bone single photon emission computed tomography imaging in evaluation of chronic low back pain: correlation with modic changes and degenerative disc disease. World Neurosurg 104:816-823, 2017

19. Tender G, Constantinescu A, Conger A, DiGiorgio A: Primary pain generator identification by CT-SPECT in a patient with low back pain: a case report. BMC Res Notes 10:132, 2017

20. Van de Kelft E, Verleye G, Van de Kelft AS, Melis K, Van Goethem J: Validation of topographic hybrid single-photon emission computerized tomography with computerized tomography scan in patients with and without nonspecific chronic low back pain. A prospective comparative study. Spine J 17:1457-1463, 2017

21. Zhong ZA, Peck A, Li S, VanOss J, Snider J, Droscha CJ, et al: ${ }^{99 m}$ TC-Methylene diphosphonate uptake at injury site correlates with osteoblast differentiation and mineralization during bone healing in mice. Bone Res 3:15013, 2015

\section{Disclosures}

The authors report no conflict of interest concerning the materials or methods used in this study or the findings specified in this paper.

\section{Author Contributions}

Conception and design: Tender, DiGiorgio. Acquisition of data: all authors. Analysis and interpretation of data: Tender, Park, DiGiorgio. Drafting the article: Tender, Davidson. Critically revising the article: Tender, Shields, Robichaux, Park, Crutcher, DiGiorgio. Reviewed submitted version of manuscript: all authors. Approved the final version of the manuscript on behalf of all authors: Tender. Statistical analysis: Tender, Shields, Robichaux, Crutcher, DiGiorgio. Administrative/technical/material support: Tender, Park, Crutcher, DiGiorgio. Study supervision: Tender.

\section{Supplemental Information \\ Previous Presentations}

Portions of this work (preliminary results) were presented in poster form at EUROSPINE, Barcelona, Spain, September 19-21, 2018.

\section{Correspondence}

Gabriel C. Tender: Louisiana State University Health Sciences Center, New Orleans, LA. gtende@1suhsc.edu. 